\title{
Traveling Waves in a Bio-reactor Model
}

\author{
Hal L. Smith* \\ Department of Mathematics \\ Arizona State University \\ Tempe, AZ 85287-1804, USA \\ E-mail: halsmith@asu.edu \\ Xiao-Qiang Zhao ${ }^{\dagger}$ \\ Department of Mathematics and Statistics \\ Memorial University of Newfoundland \\ St. John's, NF A1C 5S7, Canada \\ E-mail: xzhao@math.mun.ca
}

\begin{abstract}
The existence of a family of traveling waves is established for a parabolic system modeling single species growth in a plug flow reactor, proving a conjecture of Kennedy and Aris [5] for a similar system. The proof uses phase plane analysis, geometric singular perturbation theory and the center manifold theorem.
\end{abstract}

Key words and phrases: Bio-reactor model, traveling waves, heteroclinic orbit, singular perturbation theory, and center manifold theorem.

2000 Mathematics subject classification: 35K55, 34C37, 92D25

\section{Introduction}

Recently, an autonomous parabolic system modeling microbial growth and competition in a flow reactor has been analyzed in [1]; the periodic case is treated in [8]. The reactor is best viewed as the portion from $x=0$ to $x=L$ of a long tube with a flow from left to right carrying fresh nutrient into the reactor at $x=0$ and carrying unused nutrient and cells out of the reactor at $x=L$. On scaling the $x$-variable, the pure culture system is

*Supported in part by NSF Grant DMS 0107160.

† Supported in part by the NSERC of Canada. 
modeled by the equations

$$
\left\{\begin{array}{l}
S_{t}=\epsilon S_{x x}-v S_{x}-f(S) u \\
u_{t}=u_{x x}-v u_{x}+(f(S)-k) u
\end{array}\right.
$$

where $S(x, t)$ and $u(x, t)$ are the concentrations of nutrient and microbial population at position $x$ and time $t$, respectively. Parameter $v \geq 0$ is the flow velocity, $k>0$ is the cell death rate, and $\epsilon>0$ is the ratio of the diffusivity of the nutrient to the random cell motility coefficient of the organism. We expect $\epsilon$ to be small (see [1]) because cell motility can be substantially larger than nutrient diffusivity. We assume that the nutrient uptake function $f$ satisfies $f(0)=0$ and $f^{\prime}>0$; a typical example being the Monod function

$$
f(S)=\frac{m S}{a+S}, \quad m>0, a>0 .
$$

Danckwerts' boundary conditions are given at $x=0$ and $x=L$ (see [1]) reflecting the assumption that fresh nutrient, at concentration $S^{0}$, enters the reactor from the left at $x=0$ and the contents of the reactor are washed out at $x=L$. This implies that there exists a "washout steady state" $u=0$ and $S=S^{0}$ where the reactor is free of organisms and the nutrient concentration matches the feed concentration $S^{0}$. If this steady state is unstable to invasion of the organism, then a second steady state, which is spatially inhomogeneous and where $u>0$, is shown to exist in [1]. Obviously, this steady state represents the survival of the organism in the bio-reactor.

If $L$ is very large and if a small local inoculum of bacteria were introduced somewhere in the middle of the reactor, initialized in its washout steady state, one might expect that two "hump-shaped" pulses of bacterial cell density would move in opposite directions, toward either end, each consuming nutrient in the front of the hump and leaving a depleted nutrient level in its wake, provided the flow velocity is not too great. In order to study this possibility, we consider an infinitely long flow reactor, where $x=-\infty$ represents the left end of the finite reactor at $x=0$ and $x=+\infty$ represents the right end at $x=L$. We focus on traveling wave solutions moving to the left, against the flow, and having the form

$$
S=S(x+c t), \quad u=u(x+c t)
$$

where we might expect that $S(z), u(z)$ satisfy

$$
S(-\infty)=S^{0}, u(-\infty)=0, S(+\infty)=S_{0}<S^{0}, u(+\infty)=0 .
$$

The equations satisfied by $S(z)$ and $u(z)$ are

$$
\left\{\begin{array}{l}
0=\epsilon S^{\prime \prime}-(v+c) S^{\prime}-f(S) u \\
0=u^{\prime \prime}-(v+c) u^{\prime}+[f(S)-k] u
\end{array}\right.
$$

Integrating the second equation over the real line, we find that

$$
0=\int_{-\infty}^{+\infty}[f(S)-k] u d z
$$


which, assuming that $u$ is positive, implies that $f\left(S^{0}\right)>k$ and $f\left(S_{0}\right)<k$. We might expect this from biological considerations because the nutrient should be sufficient for growth upstream of the pulse and be depleted below the level at which bacteria can grow downstream of the pulse.

Note that the ODE reaction system corresponding to (1.1) has a one dimensional manifold $\{(S, 0): S \geq 0\}$ of equilibria. Therefore, we may expect a family of traveling waves parameterized by $S^{0}$ where $f\left(S^{0}\right)>k$ and we need to determine how $S_{0}$ depends on $S^{0}$. On adding the two equations of (1.4), integrating over the real line, and employing (1.3), we get

$$
(v+c)\left(S^{0}-S_{0}\right)=k \int_{-\infty}^{+\infty} u(z) d z
$$

relating the total biomass in the bio-reactor to the consumed nutrient $S^{0}-S_{0}$.

Equation (1.5) was derived by Kennedy and Aris [5] who considered an analogous bio-reactor model with no advection term (i.e. $v=0$ ) and sought waves moving to the right. Obviously, the advection term presents no significant complication in so far as our interest is in traveling wave solutions for (1.1). Kennedy and Aris showed the existence of traveling waves for the case $v=0$ and $\epsilon=0$ and conjectured the existence of such waves when $\epsilon>0$. We will give a proof of this conjecture for small positive $\epsilon$. It should be mentioned that Kennedy and Aris also consider traveling waves for a chemotaxis model and consider the case $k=0$ which we do not treat.

The organization of this paper is as follows. In section 2, we prove the existence of a family of traveling wave solutions for the case $\epsilon=0$ by using the first integral of the resulting three dimensional ODE system and the method of phase plane analysis. This proof is quite similar to the one given by Kennedy and Aris in [5] for the case $v=0$ but we need it and certain estimates for the case where $\epsilon>0$. In section 3, we establish the existence of a family of traveling wave solutions for small $\epsilon>0$ which are perturbations of the corresponding traveling wave solutions obtained in the case $\epsilon=0$. Geometrical singular perturbation theory and the center manifold theory play a major role in the proof.

Traveling wave solutions have been widely investigated for nonlinear reaction-diffusion equations modeling a variety of physical and biological phenomena. The literature on these solutions is vast; the reader may consult e.g., $[5,6,9,7]$, and references therein.

\section{The unperturbed system $\epsilon=0$}

In this section, we discuss the existence of traveling waves for the system (1.1) with $\epsilon=0$. Kennedy and Aris already established the existence of such waves in [5] but we provide the (slightly different) argument here in order to be able to use the result and notation later for the case $\epsilon>0$. For easy reference, (1.1) is given below for the case 
$\epsilon=0$ :

$$
\left\{\begin{array}{l}
S_{t}=-v S_{x}-f(S) u \\
u_{t}=u_{x x}-v u_{x}+(f(S)-k) u .
\end{array}\right.
$$

We are interested in non-negative traveling wave solutions of (2.1) of the form (1.2) satisfying (1.3). The condition $S^{0}>S_{0}$ ensures that we do not consider the trivial constant solutions $S \equiv$ constant, $u \equiv 0$.

It is easy to see that no such wave can exist unless $f(\infty)>k$ so we define $S^{*}$ as the unique solution of $f(S)-k=0$ and

$$
F(S)=\int_{S^{*}}^{S} \frac{1}{f(s)} d s, \quad S>0 .
$$

It follows that $(S(z), u(z))$ satisfies (1.4) which now becomes

$$
\left\{\begin{array}{l}
C S^{\prime}=-f(S) u \\
C u^{\prime}=u^{\prime \prime}+f(S) u-k u
\end{array}\right.
$$

where $C=c+v$ and hence,

$$
C u^{\prime}(z)=u^{\prime \prime}(z)-C S^{\prime}(z)+k C \frac{d}{d z} F(S) .
$$

Integrating (2.3), we get

$$
C u(z)=u^{\prime}(z)-C S(z)+k C F(S(z))+A
$$

where $A$ is a constant to be determined. Letting $z \rightarrow \pm \infty$ in (2.4), respectively, and using (1.3), we find that $S=S_{0}, S^{0}$ satisfy

$$
A / C=S-k F(S) \equiv G(S)
$$

The function $G$ satisfies

$$
G(0+)=+\infty, G(+\infty)=+\infty, G\left(S^{*}\right)=S^{*}, G^{\prime}(S)=\frac{f(S)-k}{f(S)}, G^{\prime \prime}(S)>0
$$

and therefore $G$ has a non-degenerate global minima at $S=S^{*}$.

We see that for each $B \equiv A / C>S^{*}$, there exists $S_{0}(B), S^{0}(B)$ satisfying $G\left(S_{0}\right)=$ $G\left(S^{0}\right)=B$ and

$$
0<S_{0}<S^{*}<S^{0}
$$

$S_{0}(B), S^{0}(B)$ are smooth functions of $B$ for $B>S^{*}$ and satisfy

$$
S_{0}(B), S^{0}(B) \rightarrow S^{*}, B \rightarrow S^{*} ; \quad S_{0}(B) \rightarrow 0, S^{0}(B) \rightarrow+\infty, B \rightarrow+\infty .
$$




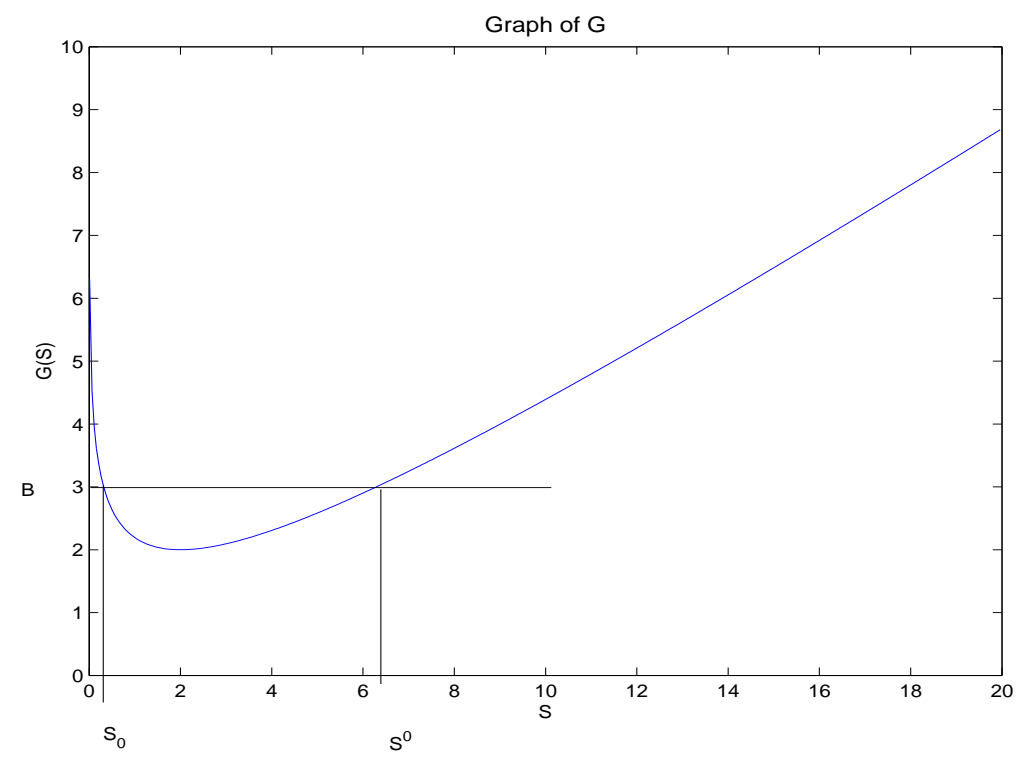

Figure 1: Horizontal line meets graph of $\mathrm{G}$

Replacing the second equation of (2.2) with equation (2.4), we then reduce the existence of traveling wave solution of (2.1) subject to (1.3) to that of heteroclinic orbit of the following two dimensional autonomous ordinary differential system

$$
\left\{\begin{array}{l}
S^{\prime}=-f(S) u / C \\
u^{\prime}=C\left[-G\left(S^{0}\right)+u+G(S)\right]
\end{array}\right.
$$

connecting the two equilibria $\left(S^{0}, 0\right)$ and $\left(S_{0}, 0\right)$ for $S^{0}>S^{*}, G\left(S_{0}\right)=G\left(S^{0}\right)$, and suitable $C>0$.

Now we are in a position to prove the main result of this section.

Theorem 2.1 For each $S^{0}>S^{*}\left(f\left(S^{*}\right)=k\right)$, there is a unique $S_{0} \in\left(0, S^{*}\right)$ satisfying

$$
G\left(S_{0}\right)=G\left(S^{0}\right) .
$$

System (2.1) has a traveling wave $(S(x+c t), u(x+c t))$ connecting $\left(S^{0}, 0\right)$ and $\left(S_{0}, 0\right)$ provided $C=c+v>0$. If $C \geq C^{*}:=\sqrt{4\left(f\left(S^{0}\right)-k\right)}$, then $S(\cdot)$ is strictly decreasing and $u(\cdot)$ is positive and unimodal; if $0<C<C^{*}$, then $(S(z), u(z))$ converges to $\left(S^{0}, 0\right)$ as $z \rightarrow-\infty$ in an oscillatory manner. Note that the wave speed $c=C-v \geq c^{*}:=C^{*}-v$ may be negative; $c^{*}>0$ if $\left.v^{2}<4\left[f\left(S^{0}\right)\right)-k\right]$.

The unimodality of $u$ means that $u^{\prime}$ vanishes once, being positive to the left and negative to the right of the zero. 
Proof. It is useful to reverse the "direction of time" in (2.7) by the change $z \rightarrow-z$ yielding

$$
\left\{\begin{array}{l}
S^{\prime}=f(S) u / C \\
u^{\prime}=C\left[G\left(S^{0}\right)-G(S)-u\right] .
\end{array}\right.
$$

Hereafter, we assume that $C>0$. We consider the domain of (2.8) to be the half space $S>0$ although we are mainly interested in certain solutions satisfying $u>0$. It has equilibria $\left(S_{0}, 0\right)$ and $\left(S^{0}, 0\right)$ where the $u^{\prime}=0$ nullcline $u_{n}(S) \equiv G\left(S^{0}\right)-G(S)$ meets the positive $S$-axis which is the $S^{\prime}=0$ nullcline. The nullcline $u_{n}(S)$ is concave down, positive on $\left(S_{0}, S^{0}\right)$ and achieves its positive maximum at $S=S^{*}$. It separates the half space $S>0$ into regions $R_{b}:=\left\{(S, u): u<u_{n}(S)\right\}$ and $R_{a}:=\left\{(S, u): u>u_{n}(S)\right\}(b$ for below, $a$ for above). We use $R_{b}^{+}$and $R_{a}^{+}$for the intersection of these sets with $u>0$.

Let $J(S, u)$ be the Jacobian matrix of the right hand side vector function of (2.8) at point $(S, u)$. It is easy to see that

$$
J(S, u)=\left[\begin{array}{cc}
\frac{1}{C} f^{\prime}(S) u & \frac{1}{C} f(S) \\
-G^{\prime}(S) C & -C
\end{array}\right] .
$$

Then the characteristic equation $\operatorname{det}(J(S, 0)-\lambda I)=0$ becomes

$$
\lambda^{2}+C \lambda+f(S)-k=0
$$

and hence, the two eigenvalues are

$$
\lambda_{ \pm}=\frac{-C \pm \sqrt{C^{2}-4(f(S)-k)}}{2} .
$$

In the case where $C^{2} \geq 4\left(f\left(S^{0}\right)-k\right)$, which we treat first, the equilibrium $\left(S^{0}, 0\right)$ of $(2.8)$ is a stable node. Since $f\left(S_{0}\right)<k$ and $C^{2} \geq 4\left(f\left(S^{0}\right)-k\right)>4\left(f\left(S_{0}\right)-k\right)$, the equilibrium $\left(S_{0}, 0\right)$ of $(2.8)$ is a saddle point. An easy calculation shows that the ratio of the components of an eigenvector $(S, U)$ of the Jacobian at $\left(S_{0}, 0\right)$ corresponding to the positive eigenvalue $\lambda_{+}$is given by $U / S=-G^{\prime}\left(S_{0}\right) \frac{C}{C+\lambda_{+}}<-G^{\prime}\left(S_{0}\right)=\left.\frac{d u_{n}}{d S}\right|_{S=S_{0}}$ and therefore the positive branch of the local unstable manifold of $\left(S_{0}, 0\right)$ enters $R_{b}^{+}$and remains there until its intersection with the boundary at $u_{n}(S)$ for some $S>S^{*}$, at which point it has a horizontal tangent line.

For $h>1$ define

$$
D_{h}=\left\{(S, u): 0 \leq u \leq h u_{n}(S), S \in\left[S_{0}, S^{0}\right]\right\}
$$

We claim that $D_{2}$ is positively invariant for system (2.8). Because of the direction field, this is trivial except along the upper boundary $u=h u_{n}(S)$, where it is easy to see that

$$
\vec{n}=-\left(h G^{\prime}(S), 1\right)
$$


is the inward normal to the curve $u=h u_{n}(S), S \in\left(S_{0}, S^{0}\right)$. It follows that on the boundary $u=h u_{n}(S), S \in\left(S_{0}, S^{0}\right)$, there holds

$$
\begin{aligned}
\left(S^{\prime}, u^{\prime}\right) \cdot \vec{n} & =-h G^{\prime}(S) f(S) u / C-C\left[u_{n}(S)-u\right] \\
& =\left(C-h G^{\prime}(S) f(S) / C\right) u-C u_{n}(S) \\
& =\left(C-h G^{\prime}(S) f(S) / C\right) h u_{n}(S)-C u_{n}(S) \\
& =\frac{u_{n}(S)}{C}\left[C^{2}(h-1)-h^{2} G^{\prime}(S) f(S)\right] \\
& =\frac{u_{n}(S)}{C}\left[C^{2}(h-1)-h^{2}(f(S)-k)\right] \\
& >\frac{u_{n}(S)}{C}\left[C^{2}(h-1)-h^{2}\left(f\left(S^{0}\right)-k\right)\right] \\
& \geq \frac{u_{n}(S)}{C}\left[4\left(f\left(S^{0}\right)-k\right)(h-1)-h^{2}\left(f\left(S^{0}\right)-k\right)\right] \\
& =\frac{u_{n}(S)}{C}\left(f\left(S^{0}\right)-k\right)\left[4(h-1)-h^{2}\right] \\
& =-\frac{u_{n}(S)}{C}\left(f\left(S^{0}\right)-k\right)(h-2)^{2} \\
& =0
\end{aligned}
$$

provided $h=2$. Note that we used $C^{2} \geq 4\left(f\left(S^{0}\right)-k\right)$ in the above calculation.

The positive branch of the local stable manifold of system $(2.8)$ at $\left(S_{0}, 0\right)$ which enters $R_{b}^{+}$and remains there until meeting the boundary at $u=u_{n}(S)$ at some $S>S^{*}$. It then enters $R_{a}^{+}$but must remain in $D_{2}$ and therefore it must converge to $\left(S^{0}, 0\right)$ as asserted. Denote by $(S(z), u(z))$ this heteroclinic orbit which is the positive branch of the unstable manifold of $\left(S_{0}, 0\right)$. As both coordinates are positive, we see that $S^{\prime}(z)>0$ and further that $u^{\prime}(z)$ has precisely one zero and $0<u(z)<u_{n}\left(S^{*}\right)=G\left(S^{0}\right)-S^{*}$.

Consequently, $(S(-z), u(-z))$ is a heteroclinic orbit of system $(2.7)$ connecting $\left(S^{0}, 0\right)$ and $\left(S_{0}, 0\right)$ with $S(-z)$ being strictly decreasing in $z \in \mathbb{R}$.

In the case that $C^{2}<4\left(f\left(S^{0}\right)-k\right)$, the equilibrium $\left(S^{0}, 0\right)$ is a stable focus. In this case, a trivial phase plane analysis shows that the positive branch of the stable manifold of system $(2.8)$ at $\left(S_{0}, 0\right)$ spirals toward $\left(S^{0}, 0\right)$ as $z \rightarrow \infty$. Indeed, this branch enters $R_{a}^{+}$as in the previous case, meets the positive $S$ axis, meets again $u=u_{n}(S)$ at $S>S^{0}$ and enters $R_{b}^{-}$, the part of $R_{b}$ with $u<0$. It cannot remain there while converging to $\left(S_{0}, 0\right)$, forming a homoclinic loop because $f(S)^{-1}$ provides a Dulac function. The corresponding assertions concerning the traveling wave solution in this case are trivial.

In the next section we will need to know how the heteroclinic orbit approaches the point $\left(S^{0}, 0\right)$.

Corollary 2.1 If $C \geq C^{*}$ then the heteroclinic orbit of (2.7), described in Theorem 
2.1, approaches $\left(S^{0}, 0\right)$ tangent to the line through that point with the slope given by

$$
\frac{\Delta u}{\Delta S}=\frac{-2\left(f\left(S^{0}\right)-k\right)}{f\left(S^{0}\right)} \cdot \frac{1}{1+(1-\chi)^{1 / 2}},
$$

where

$$
\chi=\frac{4\left(f\left(S^{0}\right)-k\right)}{C^{2}} .
$$

Proof. The solution approaches $\left(S^{0}, 0\right)$ tangent to the eigenvector corresponding to the eigenvalue $\lambda_{+}$. An easy calculation of the eigenvector leads to its slope:

$$
\frac{\Delta u}{\Delta S}=-G^{\prime}\left(S^{0}\right) \frac{C}{C+\lambda_{+}}
$$

which is precisely (2.11).

Figures 2 and 3 depict the heteroclinic orbit and time-series respectively in the case that $f(S)=2 S /(2+S), k=1, C=4$. As it turns out to be easier to compute using the "time reversed" system (2.8), the time series depicted in Figure 3 is moving in the opposite direction; the wave form for (2.7) is obtained by reflection $z \rightarrow-z$.

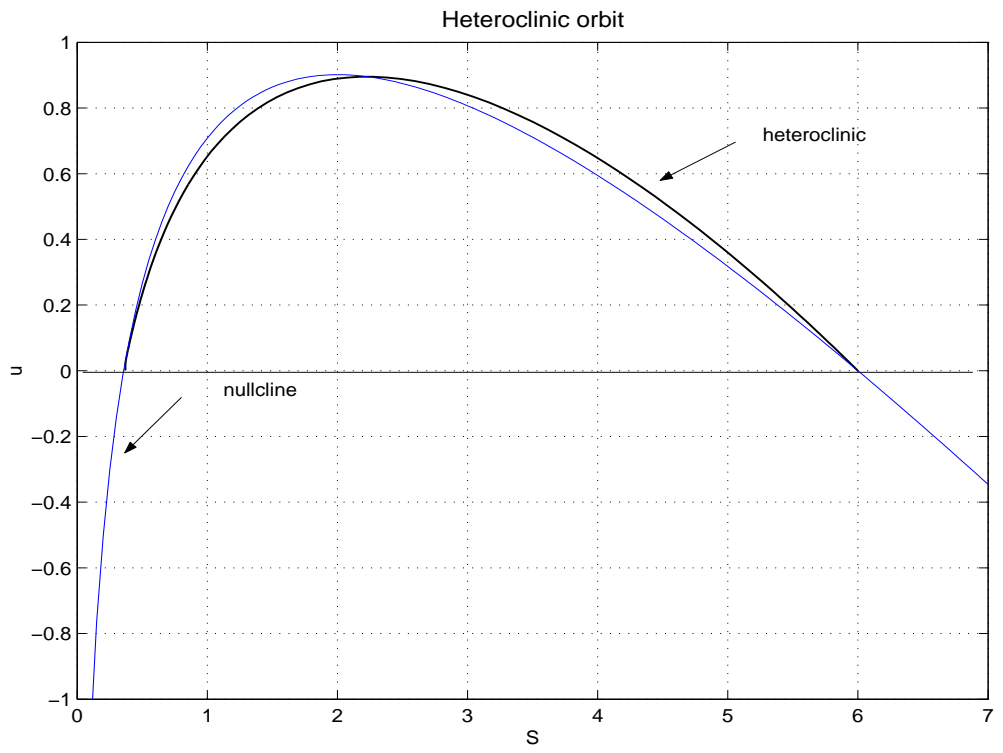

Figure 2: Heteroclinic orbit (black) and $u^{\prime}=0$ nullcline (blue) for $(2.7)$ 


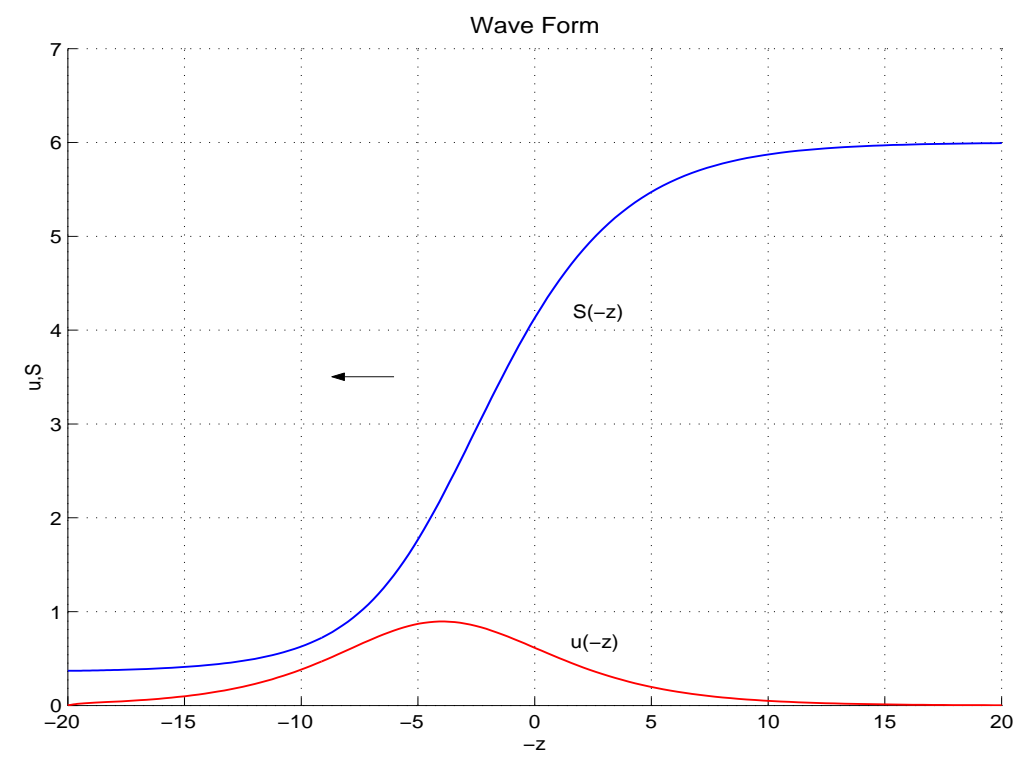

Figure 3: wave form for (2.7)-direction reversed

\section{The perturbed system}

In this section, we discuss the existence of traveling wave solutions of (1.1) for small $\epsilon>0$. On setting $C=c+v$ and letting

$$
u_{1}(z)=u(z), u_{2}(z)=u^{\prime}(z), S_{1}(z)=S(z), S_{2}(z)=S^{\prime}(z)
$$

(1.4) can be written as

$$
\left\{\begin{aligned}
u_{1}^{\prime} & =u_{2} \\
u_{2}^{\prime} & =C u_{2}-f\left(S_{1}\right) u_{1}+k u_{1} \\
S_{1}^{\prime} & =S_{2} \\
\epsilon S_{2}^{\prime} & =C S_{2}+f\left(S_{1}\right) u_{1}
\end{aligned}\right.
$$

which we call the "slow system". We must also have

$$
u_{i}( \pm \infty)=0, S_{2}( \pm \infty)=0, S_{1}(-\infty)=S^{0}, S_{1}(+\infty)=S_{0}
$$

where $S_{0}<S^{*}<S^{0}$.

The corresponding fast system is obtained by the change of dependent variable $\eta=$ $z / \epsilon($ dot denotes derivative with respect to $\eta)$ :

$$
\left\{\begin{array}{l}
\dot{u}_{1}=\epsilon u_{2} \\
\dot{u}_{2}=\epsilon\left[C u_{2}-f\left(S_{1}\right) u_{1}+k u_{1}\right] \\
\dot{S}_{1}=\epsilon S_{2} \\
\dot{S}_{2}=C S_{2}+f\left(S_{1}\right) u_{1}
\end{array}\right.
$$


Denote by

$$
E=\left\{\left(0,0, S_{1}, 0\right): S_{1} \geq 0\right\}
$$

the one dimensional manifold of equilibria for (3.1). The linearization of the slow system about a point of $E$ gives

$$
\left[\begin{array}{cccc}
0 & 1 & 0 & 0 \\
-f\left(S_{1}\right)+k & C & 0 & 0 \\
0 & 0 & 0 & 1 \\
f\left(S_{1}\right) / \epsilon & 0 & 0 & C / \epsilon
\end{array}\right]
$$

The eigenvalues coincide with those of the two-by-two submatrices on the diagonal; eigenvalues of the top left two-by-two matrix are

$$
\lambda_{ \pm}=\frac{C}{2} \pm \frac{\sqrt{C^{2}-4 r}}{2}
$$

where $r=f\left(S_{1}\right)-k$ and are negatives of those in (2.10). These are real provided $C^{2}>4 r$. We see that there is exactly one zero eigenvalue, except at the point $\left(0,0, S^{*}, 0\right) \in E$. Points of $E$ with $S_{1}>S^{*}$ have 3 eigenvalues with positive real part while points with $S_{1}<S^{*}$ have 2 positive eigenvalues and one negative one. Away from $\left(0,0, S^{*}, 0\right), E$ is normally hyperbolic.

Consider the fast system. For $\epsilon=0$ we have the three dimensional manifold of equilibria given by

$$
M_{0}=\left\{\left(u_{1}=u_{1}^{0}, u_{2}=u_{2}^{0}, S_{1}=S_{1}^{0}, S_{2}=-f\left(S_{1}^{0}\right) u_{1}^{0} / C\right\} .\right.
$$

$E \subset M_{0}$ and an easy calculation shows that the eigenvalues of the Jacobian, on setting $\epsilon=0$, has 3 zero eigenvalues corresponding to the tangent space of $M_{0}$ and one positive eigenvalue, namely $C$. Thus, $M_{0}$ is normally hyperbolic for $C>0$ (see [4] for definition).

According to Fenichel's Invariant Manifold Theorem(see [3, 4]), for $0 \leq \epsilon<\epsilon_{0}$ there exists a locally invariant manifold

$$
M_{\epsilon}=\left\{\left(u_{1}=u_{1}^{0}, u_{2}=u_{2}^{0}, S_{1}=S_{1}^{0}, S_{2}=-f\left(S_{1}^{0}\right) u_{1}^{0} / C+\epsilon h\left(u_{1}^{0}, u_{2}^{0}, S_{1}^{0}, \epsilon\right)\right\}\right.
$$

for the fast system, where $h$ is a smooth function defined on a compact domain. The dynamics on $M_{\epsilon}$ are given by (we have changed back to slow time)

$$
\left\{\begin{array}{l}
u_{1}^{\prime}=u_{2} \\
u_{2}^{\prime}=C u_{2}-f\left(S_{1}\right) u_{1}+k u_{1} \\
S_{1}^{\prime}=-f\left(S_{1}\right) u_{1} / C+\epsilon h\left(u_{1}, u_{2}, S_{1}, \epsilon\right) .
\end{array}\right.
$$

Note that because of the manifold of equilibria $E, h$ must satisfy $h\left(0,0, S_{1}, \epsilon\right)=0$ for all $S_{1}$. 
When $\epsilon=0$, the flow on $M_{0}$ is given by

$$
\left\{\begin{array}{l}
u_{1}^{\prime}=u_{2} \\
u_{2}^{\prime}=C u_{2}-f\left(S_{1}\right) u_{1}+k u_{1} \\
S_{1}^{\prime}=-f\left(S_{1}\right) u_{1} / C
\end{array}\right.
$$

According to Theorem 2.1, for $0<S_{0}<S^{*}$ and $C>0$, the positive branch of the onedimensional stable manifold of $\left(0,0, S_{0}\right)$ for system $(3.4), W_{0}^{s}\left(S_{0}\right)$, connects to $\left(0,0, S^{0}\right)$, where $G\left(S_{0}\right)=G\left(S^{0}\right)>S^{*}$. Note that system (3.4) is equivalent to (2.2). We want to show that for $\epsilon>0$ but very small, the positive branch of the one-dimensional stable manifold of $\left(0,0, S_{0}\right)$ for system $(3.3), W_{\epsilon}^{s}\left(S_{0}\right)$, connects to some point $(0,0, \hat{S})$ near $\left(0,0, S^{0}\right)$. Although the global stable manifold does not perturb nicely, "compact pieces" of it do because the local stable manifold depends continuously on $\epsilon$ and because of continuous dependence of solutions over finite time intervals on parameters. To be more precise, we may describe the local stable manifold as the forward orbit $\left\{x_{\epsilon}(t)\right.$ : $t \geq 0\}$ of (3.3) through a point $x_{\epsilon}:=x_{\epsilon}(0)$ on the local stable manifold, which depends continuously on $\epsilon$, and by a compact piece of the global stable manifold we mean $\left\{x_{\epsilon}(t)\right.$ : $t \geq-T\}(T \gg 1)$, with endpoint $x_{\epsilon}(-T)$. We expect that such a compact piece of $W_{\epsilon}^{s}\left(S_{0}\right)$ has endpoint nearby $\left(0,0, S^{0}\right)$ for small $\epsilon>0$. The next result indicates what happens to the backward orbit through this endpoint.

Lemma 3.1 Given $S^{0}>S^{*}$ and $\delta_{0}>0$, there exists $\epsilon_{0}, \delta>0$ such that if $p=\left(u_{1}, u_{2}, S_{1}\right)$ satisfies $\left|p-\left(0,0, S^{0}\right)\right|<\delta$ and $0 \leq \epsilon<\epsilon_{0}$, then the solution of (3.3) starting at $p$, $x^{\epsilon}(t)=\left(u_{1}^{\epsilon}(t), u_{2}^{\epsilon}(t), S_{1}^{\epsilon}(t)\right)$, satisfies $\left|x^{\epsilon}(t)-\left(0,0, S^{0}\right)\right|<\delta_{0}$ for all $t<0$ and there exists $q_{\epsilon}=\left(0,0, S_{\epsilon}\right)$ such that $x^{\epsilon}(t) \rightarrow q_{\epsilon}$ as $t \rightarrow-\infty$.

Proof. We apply the center manifold theory in [2] to the time reversed system (3.3) with the equation $\epsilon^{\prime}=0$ appended to it. Note that this four dimensional system has the two dimensional manifold of equilibria given by $N=\left\{u_{1}=u_{2}=0\right\}$. Focus on one of these steady states $Z \equiv\left(u_{1}, u_{2}, S_{1}, \epsilon\right)=\left(0,0, S^{0}, 0\right)$. A change of variables $S_{1} \rightarrow v_{1}$, given by

$$
v_{1}=S_{1}-S^{0}+\left(f\left(S^{0}\right) / r\right)\left[u_{1}-u_{2} / C\right], \quad r=f\left(S^{0}\right)-k
$$

translates $Z$ to the origin and de-couples the linear parts of the time reversed system (3.3). The resulting system is given by

$$
\left\{\begin{array}{l}
u_{1}^{\prime}=-u_{2} \\
u_{2}^{\prime}=r u_{1}-C u_{2}+\left[f\left(S_{1}\right)-f\left(S^{0}\right)\right] u_{1} \\
v_{1}^{\prime}=-\frac{k}{C r}\left[f\left(S_{1}\right)-f\left(S^{0}\right)\right] u_{1}-\epsilon h\left(u_{1}, u_{2}, S_{1}, \epsilon\right) \\
\epsilon^{\prime}=0
\end{array}\right.
$$

where $S_{1}$ is determined by (3.5). If we let $x=\left(v_{1}, \epsilon\right)$ and $y=\left(u_{1}, u_{2}\right)$, then this system 
has the form

$$
\left\{\begin{array}{l}
x^{\prime}=A x+f(x, y) \\
y^{\prime}=B y+g(x, y)
\end{array}\right.
$$

where $A$ is the zero matrix and

$$
B=\left[\begin{array}{ll}
0 & -1 \\
r & -C
\end{array}\right]
$$

is a stable matrix. $f(x, y)$ and $g(x, y)$ are higher order terms in the sense that their first derivatives vanish at the origin. [2, Theorem 1] asserts the existence of a center manifold at the origin for (3.6) but we already know this. In fact, the center manifold (its unique in our case) is just the manifold of equilibria $N$. The dynamics on $N$ is trivial:

$$
\left\{\begin{aligned}
v_{1}^{\prime} & =0 \\
\epsilon^{\prime} & =0 .
\end{aligned}\right.
$$

Since our equilibrium $v_{1}=0, \epsilon=0$ is stable for the dynamics on $N$, [2, Theorem 2] implies that the origin is Lyapunov stable for (3.6). Furthermore, by the second assertion of $\left[2\right.$, Theorem 2], a solution $\left(u_{1}(t), u_{2}(t), v_{1}(t), \epsilon\right)$ of $(3.6)$ which starts near the origin satisfies

$$
u_{i}(t)=O\left(e^{-\gamma t}\right), v_{1}(t)=v_{1}^{0}+O\left(e^{-\gamma t}\right), t \rightarrow \infty
$$

where $\gamma>0$ and $v_{1}^{0}$ is small. Thus, we get $S_{1}(t)=S^{0}+v_{1}^{0}+O\left(e^{-\gamma t}\right)$. This is exactly what we assert in Lemma 3.1.

Now we are ready to prove the main results in this section.

Theorem 3.1 Let $S_{0}$ satisfy $0<S_{0}<S^{*}$ and let $S^{0}>S^{*}$ satisfy $G\left(S_{0}\right)=G\left(S^{0}\right)$. If $\epsilon>0$ is sufficiently small and $C=c+v>0$, then system (1.1) has a unique (up to translation) traveling wave solution $(S(z), u(z))(z=x+c t)$ connecting $(\hat{S}, 0)$ and $\left(S_{0}, 0\right)$ with $u(z)>0$ for $z \approx+\infty$, where $\hat{S}=\hat{S}(\epsilon)>S^{*}$ is near $S^{0}$ and $\hat{S}(\epsilon) \rightarrow S^{0}$ as $\epsilon \rightarrow 0$. Indeed, $S(z)=S_{1}(z), u(z)=u_{1}(z)$ where $\left(u_{1}(z), u_{2}(z), S_{1}(z)\right)$ is a solution of (3.3) representing the branch of the one-dimensional stable manifold $W_{\epsilon}^{s}\left(S_{0}\right)$ of $\left(0,0, S_{0}\right)$ for which $u(z)>0$ near $+\infty$.

Proof. For $0<S_{0}<S^{*}$, "compact pieces" of the positive branch of the onedimensional stable manifold of $\left(0,0, S_{0}\right)$ for $(3.3), W_{\epsilon}^{s}\left(S_{0}\right)$, are $O(\epsilon)$ close to corresponding pieces of $W_{0}^{s}\left(S_{0}\right)$. But $W_{0}^{s}\left(S_{0}\right)$ connects $\left(0,0, S_{0}\right)$ to $\left(0,0, S^{0}\right)$ by Theorem 2.1. If $S^{0}$ satisfies $G\left(S_{0}\right)=G\left(S^{0}\right), \delta_{0}>0$ is chosen smaller than $\left(S^{0}-S^{*}\right) / 2$, and $\delta>0$ is as in Lemma 3.1, then there exists $\epsilon_{1}>0$ such that for all $\epsilon \in\left[0, \epsilon_{1}\right)$, a compact piece of $W_{\epsilon}^{s}\left(S_{0}\right)$ has end point within distance $\delta$ of $\left(0,0, S^{0}\right)$. We can assume that $\epsilon_{1}<\epsilon_{0}$ of Lemma 3.1 and so, according to Lemma 3.1, the backward continuation of the compact 
piece of $W_{\epsilon}^{s}\left(S_{0}\right)$ is asymptotic to a point $q_{\epsilon}=\left(0,0, S_{\epsilon}\right)$ satisfying $\left|q_{\epsilon}-\left(0,0, S^{0}\right)\right| \leq \delta_{0}$. Thus, we have shown the existence of a heteroclinic orbit for $(3.3)$ connecting $\left(0,0, S_{0}\right)$ to $q_{\epsilon}$. Consequently, there exists a heteroclinic orbit of $(3.1)$ connecting $(0,0, \hat{S}, 0)$ to $\left(0,0, S_{0}, 0\right)$ where $q_{\epsilon}=(0,0, \hat{S})$.

Some comments concerning Theorem 3.1 will be helpful. First, we note that the traveling wave solution described in the theorem depends on both $\epsilon$ and on $C$, a fact not reflected in our notation for simplicity. Second, it should be observed that we make no assertions about the signs of $u_{1}$ or $S_{1}^{\prime}$. In Theorem 3.2 below, we take up these issues.

An estimate of $\hat{S}$ can be made following Kennedy and Aris [5], who derived it assuming the existence of such a wave. From the first of equations (1.4) we find that

$$
u=\frac{\epsilon S^{\prime \prime}-C S^{\prime}}{f(S)}
$$

which, on substitution into (1.5) and integration by parts, leads to

$$
G(\hat{S})=G\left(S_{0}\right)+\frac{k \epsilon}{C} \int_{-\infty}^{+\infty} \frac{f^{\prime}(S)}{f^{2}(S)}\left(S^{\prime}(z)\right)^{2} d z
$$

where $G$ is defined by $(2.5)$ and $S(z)$ is the component of the traveling wave solution given in Theorem 3.1. Recalling that $S^{0}$ is defined by $G\left(S^{0}\right)=G\left(S_{0}\right)$ and that $G$ is increasing for $S>S^{*}$, it follows from the positivity of the integral on the right that $\hat{S}(\epsilon)>S^{0}$. Equation (3.7) also allows the calculation of the order $\epsilon$ term in a Taylor expansion of $\hat{S}(\epsilon)$ about $\epsilon=0$.

Theorem 3.2 Let $S_{0}$ satisfy $0<S_{0}<S^{*}$ and $C=c+v>C^{*}:=\sqrt{4\left(f\left(S^{0}\right)-k\right)}$. If $\epsilon$ is sufficiently small, then the traveling wave solution described in Theorem 3.1, $(S(x+c t), u(x+c t))$, has the property that $S(\cdot)$ is strictly decreasing and $u(\cdot)$ is positive and unimodal. The wave speed $c=C-v$ may be negative.

Proof. For $\epsilon=0$ we know that $u_{1}(z)=u(z), u_{2}(z)=u^{\prime}(z)$ satisfies (see 2.7)

$$
\frac{u^{\prime}(z)}{u(z)}=-\frac{u^{\prime}(z)}{S^{\prime}(z)} \frac{f(S(z))}{C}
$$

Letting $z \rightarrow-\infty$, we see from (2.11) that this ratio approaches

$$
\frac{2 r^{0}}{C} \frac{1}{1+(1-\chi)^{1 / 2}}<\frac{2 r^{0}}{C}<\frac{C}{2}
$$

where we use $C^{2} / 4>r^{0}=f\left(S^{0}\right)-k$ in the last inequality. If $T$ is sufficiently large, then $u(-T), u^{\prime}(-T)>0$ and

$$
\frac{u^{\prime}(-T)}{u(-T)}<\frac{C}{2}
$$


By continuity, for $\epsilon>0$ sufficiently small, we have that $u_{1}>0$ along the part of the heteroclinic orbit which lies outside the small $\delta$-neighborhood of $\left(0,0, S^{0}\right)$ identified in Lemma 3.1. By choosing $T$ larger if necessary, we can assume that $\left(u_{1}(z), u_{2}(z), S_{1}(z)\right)$ belongs to the $\delta$-neighborhood of $\left(0,0, S^{0}\right)$ for $z \leq-T$, that $u_{1}(z)>0$ for $-T \leq z<\infty$, $u_{2}(-T)>0$ and that

$$
\frac{u_{2}(-T)}{u_{1}(-T)}<\frac{C}{2}
$$

We wish to show that $u_{1}(z)>0$ for all $z$. Therefore, it is only necessary to consider $\left(u_{1}(z), u_{2}(z), S_{1}(z)\right)$ for $z \leq-T$.

For this it will be easier to follow if we reverse "time" by setting $\bar{z}=-z$ as in the proof of Theorem 2.1. Then we need to consider the heteroclinic orbit $\left(u_{1}(\bar{z}), u_{2}(\bar{z}), S_{1}(\bar{z})\right)$ for $\bar{z} \geq T$, which belongs to the $\delta$-neighborhood of $\left(0,0, S^{0}\right)$. Hereafter, we drop the bar over $z$.

Now we change dependent variables in $(3.3)$ by replacing $\left(u_{1}, u_{2}\right)$ by polar coordinates $(\rho, \theta)$. The result, after some algebra and accounting for the change $z \rightarrow-z$, is:

$$
\left\{\begin{aligned}
\rho^{2} \theta^{\prime} & =-C u_{1} u_{2}+r u_{1}^{2}+u_{2}^{2} \\
& =-\left(\frac{C^{2}}{4}-r\right) u_{1}^{2}+\left(u_{2}-C u_{1} / 2\right)^{2} \\
\rho \rho^{\prime} & =-(1-r) u_{1} u_{2}-C u_{2}^{2} \\
S_{1}^{\prime} & =f\left(S_{1}\right) u_{1} / C-\epsilon h\left(u_{1}, u_{2}, S_{1}, \epsilon\right) .
\end{aligned}\right.
$$

where $r=f\left(S_{1}\right)-k$ depends on $S_{1}(z)$. We have not converted the right hand side to polar coordinates in order to make an argument later on. We are interested in (3.9) for $z \geq T$ where $S_{1}(z)-S^{0}$ is so small that $C^{2} / 4-r>0$. By (3.8) we see that $\left(u_{1}(T), u_{2}(T)\right)$ belongs to the open first quadrant and that

$$
0<\theta(T)=\tan ^{-1}\left(\frac{u_{2}(T)}{u_{1}(T)}\right)<\theta_{0}:=\tan ^{-1}\left(\frac{C}{2}\right) .
$$

By the first equation of (3.9), it easily follows that $\theta^{\prime}(z)>0$ whenever $\theta(z)=0$ (i.e., $u_{2}=$ 0 ) and $\theta^{\prime}(z)<0$ whenever $\theta(z)=\theta_{0}$ (i.e., $u_{2}=\frac{C}{2} u_{1}$ ). Thus, $0 \leq \theta(z) \leq \theta_{0}$ for all $z \geq T$ and, in particular, $u_{1}(z)>0$ for $z \geq T$. We have established that $u_{1}(z)>0$ for all $z$.

A simple computation shows that the vector

$$
\vec{V}:=\left(1, \lambda_{-}, \frac{-f\left(S_{0}\right)}{\lambda_{-}\left(c-\epsilon \lambda_{-}\right)}, \frac{-f\left(S_{0}\right)}{c-\epsilon \lambda_{-}}\right)
$$

is an eigenvector of the Jacobian matrix of the original system $(3.1)$ at $\left(0,0, S_{0}, 0\right)$ associated with the negative eigenvalue $\lambda_{-}$given by (3.2) with $r=f\left(S_{0}\right)-k<0$. Since the heteroclinic orbit $\left(u_{1}(z), u_{2}(z), S_{1}(z), S_{2}(z)\right)$ tends to $\left(0,0, S_{0}, 0\right)$ in the direction of $\vec{V}$ as $z \rightarrow+\infty$, the third equation of (3.1) implies that $S_{1}^{\prime}(z)<0$ when $z$ is very close to 
$+\infty$. We further claim that $S_{1}^{\prime}(z)$ has no zero on $\mathbb{R}$. Otherwise, $S_{1}^{\prime}(z)$ admits a largest zero $z_{0}$ on $\mathbb{R}$ and

$$
\epsilon S_{1}^{\prime \prime}\left(z_{0}\right)=C S_{1}^{\prime}\left(z_{0}\right)+f\left(S_{1}\left(z_{0}\right)\right) u_{1}\left(z_{0}\right)=f\left(S_{1}\left(z_{0}\right)\right) u_{1}\left(z_{0}\right)>0 .
$$

Then there exists a $z_{1}>z_{0}$ such that $S_{1}^{\prime}\left(z_{1}\right)>0$, and hence, there exists $z_{2} \in\left(z_{1},+\infty\right)$ such that $S_{1}^{\prime}\left(z_{2}\right)=0$, a contradiction. Thus, $S_{1}^{\prime}(z)<0$ for all $z \in \mathbb{R}$.

Note that the second component of $\vec{V}$ is negative. Then $u_{2}(z)$ is negative when $z$ is very close to $+\infty$. Since $u_{1}( \pm \infty)=0$ and $u_{1}^{\prime}(z)=u_{2}(z), u_{2}(z)$ admits at least one zero. By the second equation of (3.1), it follows that

$$
u_{2}^{\prime}(z)=\left[k-f\left(S_{1}(z)\right)\right] u_{1}(z) \quad \text { whenever } \quad u_{2}(z)=0 .
$$

Let $y_{0}$ be the largest zero of $u_{2}(z)$. Then $u_{2}^{\prime}\left(y_{0}\right) \leq 0$ and $u_{2}(z)<0, \forall z>y_{0}$. Letting $z=y_{0}$ in (3.10), we have $S_{1}\left(y_{0}\right) \geq S^{*}$. Since $S_{1}(z)$ is strictly decreasing on $\mathbb{R}$, there holds $S_{1}(z)>S^{*}, \forall z<y_{0}$. We claim that $u_{2}^{\prime}\left(y_{0}\right)<0$. Suppose that $u_{2}^{\prime}\left(y_{0}\right)=0$. Then the second equation of (3.1) implies that $u_{2}^{\prime \prime}\left(y_{0}\right)=-f^{\prime}\left(S_{1}\left(y_{0}\right)\right) S_{1}^{\prime}\left(y_{0}\right) u_{1}\left(y_{0}\right)>0$, and hence $u_{2}\left(y_{0}\right)=0$ is the local minimum of $u_{2}(z)$ around $y_{0}$, which contradicts the choice of $y_{0}$. Thus, $u_{2}(z)$ changes sign at $y_{0}$. We further claim that $u_{2}(z)$ admits no zero in $\left(-\infty, y_{0}\right)$. Otherwise, let $y_{1}$ be the largest zero of $u_{2}(z)$ in $\left(-\infty, y_{0}\right)$. Then we have $u_{2}^{\prime}\left(y_{1}\right) \geq 0$. But (3.10) with $z=y_{1}$ implies that $S_{1}\left(y_{1}\right) \leq S^{*}$, a contradiction. It follows that $u_{1}^{\prime}(z)=u_{2}(z)>0, \forall z \in\left(-\infty, y_{0}\right)$ and $u_{1}^{\prime}(z)=u_{2}(z)<0, \forall z \in\left(y_{0},+\infty\right)$, and hence $u_{1}(z)$ is positive and unimodal.

\section{References}

[1] M. Ballyk, D. Le, D. A. Jones and H. L. Smith, Effects of random motility on microbial growth and competition in a flow reactor, SIAM J. Appl. Math., 59(1999), 573-596.

[2] J. Carr, "Applications of Centre Manifold Theory", Applied Mathematical Sciences, Vol. 35, Springer-Verlag, New York, 1981.

[3] N. Fenichel, Geometrical singular perturbation theory for ordinary differential equations, Journal of Differential Equations, 31(1979), 53-98.

[4] C. K. R. T. Jones, Geometrical singular perturbation theory, in "Dynamical Systems" (R. Johnson, Ed.), Lecture Notes in Mathematics, Vol. 1609, Springer-Verlag, New York, 1995.

[5] C. R. Kennedy and R. Aris, Traveling waves in a simple population model involving growth and death, Bulletin of Mathematical Biology, 42(1980), 397-429.

[6] J. D. Murray, "Mathematical Biology", Springer-Verlag, New York, 1989. 
[7] V. Rottschafer and C. E. Wayne, Existence and stability of traveling fronts in the extended Fisher-Kolmogorov equation, Journal of Differential Equations, 176(2001), 532-560.

[8] H. L. Smith and X.-Q. Zhao, Dynamics of a periodically pulsed bio-reactor model, Journal of Differential Equations, 155(1999), 368-404.

[9] A. I. Volpert, Vitaly A. Volpert and Vladimir A. Volpert, "Traveling Wave Solutions of Parabolic Systems", Transl. Math. Monogr. 140, AMS, Providence, RI, 1994. 